\title{
Oral Administration of Herbal Mixture Extract Inhibits 2,4-Dinitrochlorobenzene-Induced Atopic Dermatitis in BALB/c Mice
}

\author{
Soon Re Kim, ${ }^{1}$ Han-Seok Choi, ${ }^{1}$ Hye Sook Seo, ${ }^{1}$ Jin Mo Ku, ${ }^{1}$ Se Hyang Hong, ${ }^{1}$ \\ Hye Hyun Yoo, ${ }^{2}$ Yong Cheol Shin, ${ }^{1}$ and Seong-Gyu Ko ${ }^{1}$ \\ ${ }^{1}$ Laboratory of Clinical Biology and Pharmacogenomics, Department of Preventive Medicine, College of Oriental Medicine, \\ Kyung Hee University, Seoul 130-701, Republic of Korea \\ ${ }^{2}$ Department of Pharmacy, College of Pharmacy, Hanyang University, Ansan, Gyeonggi-do 426-791, Republic of Korea
}

Correspondence should be addressed to Seong-Gyu Ko; epiko@khu.ac.kr

Received 17 February 2014; Accepted 10 June 2014; Published 16 July 2014

Academic Editor: Chang Shik Yin

Copyright (C) 2014 Soon Re Kim et al. This is an open access article distributed under the Creative Commons Attribution License, which permits unrestricted use, distribution, and reproduction in any medium, provided the original work is properly cited.

\begin{abstract}
CP001 is four traditional herbal medicine mixtures with anti-inflammatory properties. In this study, we investigated the effect of oral administration of CP001 ethanol extract on the 2,4-dinitrochlorobenzene- (DNCB-) induced AD mouse models. For that purpose, we observed the effects of oral administration of CP001 on skin inflammatory cell infiltration, skin mast cells, production of serum IgE, and expression of Th2 cytokine mRNA in the AD skin lesions of DNCB treated BALB/c mice. Histological analyses demonstrated that CP001 decreased dermis and epidermis thickening as well as dermal infiltration induced by inflammatory cells. In addition, CP001 decreased mast cell infiltration in count as well as dermal infiltration induced by inflammatory cells. In the skin lesions, mRNA expression of interleukin- (IL-) 4 and IL-13 was inhibited by CP001. CP001 also reduced the production of IgE level in mouse plasma. In addition, we investigated the effect of CP001 on the inflammatory allergic reaction using human mast cells (HMC-1). In HMC-1, cytokine production and mRNA levels of IL-4, IL-13, IL-6, and IL-8 were suppressed by CP001. Taken together, our results showed that oral administration of CP001 exerts beneficial effects in AD symptoms, suggesting that CP001 might be a useful candidate for the treatment of $\mathrm{AD}$.
\end{abstract}

\section{Introduction}

Atopic dermatitis (AD) is a most common chronic inflammatory skin disease, affecting about 10 million people in the world, leading to a significant reduction in quality of life, and its incidence is continuously increasing in westernized countries $[1,2]$. The pathogenesis of $\mathrm{AD}$ is unknown, but the disease seems to be correlated with specific immune and inflammatory mechanisms. The general characteristics of AD include excessive infiltration of inflammatory cells such as lymphocytes, macrophages, and granulated mast cells into the skin lesions, eosinophilia in peripheral blood, and a high level of serum immunoglobulin E (IgE) [3].

Mast cells are tissue-based inflammatory cells of bone marrow origin, which respond to signals of innate and adaptive immunity. They play a major role in immediate hypersensitivity reaction and are activated through the high-affinity IgE receptor, FceR [4]. In addition, it has been reported that a large number of mast cells can be found in $\mathrm{AD}$ skin lesion. The majority of AD patients have elevated blood IgE level and it is mediated of mast cell activation [5]. Mast cell activation by $\operatorname{IgE}$ is release inflammatory mediators, such as histamine, as well as cytokines, including Th2 cytokines, such as IL-4, IL-5, and IL-13. Thus, cell-bound IgE is crosslinked allergens and it is contributed to the development of $\mathrm{AD}$ through mediating activation of mast cells localization [6].

Proinflammatory cytokines that are released by activated mast cells, including IL- 6 and IL-8, play an important role in allergic inflammation [7]; IL-6 mediates allergic inflammation [8], while IL-8 induces the migration of neutrophils into inflammatory regions as a potent chemotactic cytokine $[9,10]$. 
CP001 is a mixture of four oriental herbal medicines composed of Houttuynia cordata Thunb, Rehmannia glutinosa Libosch, bark of Betula platyphylla var. japonica, and Rubus coreanus Miq. Houttuynia cordata Thunb has long been used in traditional oriental medicine for the treatment of inflammatory diseases. Also, several studies demonstrated that Houttuynia cordata Thunb has been associated with a broad range of pharmacological activities, including antiinflammatory [1], antiviral [11], and anticancer effects [12]. Rehmannia glutinosa Libosch has traditionally been used as an ingredient herb in East Asian medicine for the effects of hemostasis, activation of blood circulation, and improvement of kidney function [13]. Several studies indicated that Rehmannia glutinosa Libosch has antiallergic effects [14] and anti-inflammatory function [15-17]. Betula platyphylla var. japonica is known to have antioxidant, anti-inflammatory, and anticancer effects [18] and inhibits the development of $\mathrm{AD}$ in $\mathrm{NC} / \mathrm{Nga}$ mice $[19,20]$. Rubus coreanus Miq. is a type of red raspberry that grows wild in Korea, Japan, and China. The fruit, known as "Bokbunja" in Korean, has been used in traditional oriental medicine for reducing the risk of diseases such as asthma and allergy. It is also known that Rubus coreanus Miq. has anti-inflammatory and antioxidative activities [21-23]. These collective observations indicate that CP001 may be good candidate for control of AD and beneficial in the treatment of human allergic disorders. Therefore, in our previous study, we already confirmed that topical application of KM110329 (CP001 modifying herbal mixture) inhibits the atopic dermatitis in ovalbumin- and DNCB-induced mouse model.

Therefore, in this study, we investigated whether $30 \%$ ethanol extract of CP001 oral administration has anti-inflammatory activity in 2,4-dinitrochlorobenzene(DNCB-) induced AD mice model. In addition, we also investigated whether $30 \%$ ethanol extract of CP001 has antiallergic effect inhibiting cytokine production in human mast cells, HMC-1.

\section{Material and Method}

2.1. Preparation of CP001. CP001 was prepared by Hanpoong Pharmaceutical (Jeon-ju, Korea) following good manufacturing practices (GMP) procedure. CP001 is 30\% ethanol extracted brown-colored powder, and it is composed of Houttuynia cordata Thunb, Rehmannia glutinosa Libosch, bark of Betula platyphylla var. japonica, and Rubus coreanus Miq. The powder from the extract was dissolved in distilled water for in vivo and in vitro experiments.

2.2. Animals. Six-week-old male BALB/c mice were purchased from Orient (Sung-nam, Korea). The mice were randomized into 6 groups (normal, DNCB, and 25, 50, 100, and $200 \mathrm{mg} / \mathrm{kg}$ (CP001)), each comprising five mice. All mice were kept under pathogen-free environment and allowed free access to the diet and water. All procedures performed on the mice were approved by the animal care center of Kyung-Hee University (Approval Number KHUASP (SE)-2012-004).

2.3. Induction of AD-Like Skin Lesions and CP001 Treatment. Induction of $\mathrm{AD}$-like skin lesions procedure is described in Figure 1. For that purpose, mice back skin was painted dermally with $200 \mu \mathrm{L}$ of a $1 \%$ DNCB using $1 \times 1 \mathrm{~cm}$ patches after shaving. Two weeks after sensitization, the back skin was challenged with $200 \mu \mathrm{L}$ of a $0.2 \%$ DNCB solution twice per week. This procedure was repeated for 2 weeks and CP001 was orally administrated together. At the end of the experiment, mice were sacrificed by $\mathrm{CO}_{2}$ inhalation, and samples were collected.

2.4. Histological Analysis. A portion of the skin biopsies were fixed in $4 \%$ paraformaldehyde (PFA) and embedded in Tissue-Tek optical cutting temperature (O.C.T.) compound (Tissue-Tek, Sakura, AA Zoeterwoude, the Netherlands) on dry ice. Skin sections of $20 \mu \mathrm{m}$ were cut and stained with hematoxylin and eosin ( $\mathrm{H} \& \mathrm{E}$ ) for inflammatory cells or with toluidine blue for mast cells counts and examined under light microscopy (Olympus). Mast cells were counted in 10 parts of high-power fields (HPF) at 400x magnification.

\subsection{Enzyme-Linked Immune Sorbent Assay. After final CP001} administration, whole blood samples were collected by cardiac puncture for measurement of blood IgE level. The blood was placed in Vacutainer tubes containing EDTA (BD science, NJ, USA) and blood plasma was isolated. Total IgE levels in plasma were determined by sandwich ELISA using the BD PharMingen mouse IgE ELISA set. Briefly, plates were coated with capture antibody in ELISA coating buffer (SigmaAldrich) and incubated overnight at $4^{\circ} \mathrm{C}$. Plates were washed with PBS-Tween $20(0.05 \%)$ and subsequently blocked $(10 \%$ FBS in PBS) for $1 \mathrm{~h}$ at $20^{\circ} \mathrm{C}$. Serial dilutions of standard antigen or sample in dilution buffer (10\% FBS in PBS) were added to the plates and plates were incubated for $2 \mathrm{~h}$ at $20^{\circ} \mathrm{C}$. After washing, biotin-conjugated anti-mouse $\operatorname{IgE}$ and SAv-HRP (streptavidin-horseradish peroxidase conjugate) were added to the plates and plates were incubated for $1 \mathrm{~h}$ at $20^{\circ} \mathrm{C}$. Finally, tetramethylbenzidine (TMB) substrate solution was added to the plates and after 15 min incubation in the dark, a $2 \mathrm{~N} \mathrm{H}_{2} \mathrm{SO}_{4}$ solution was added to stop the reaction. Optical densities were measured at $450 \mathrm{~nm}$ on an automated ELISA reader (Versa Max, Molecular Devices, CA, USA). IL-6 and IL-8 levels were measured in HMC1 supernatant by sandwich ELISA using BD Pharmingen human ELISA set. The sandwich ELISA procedures were performed by following the same protocols described above.

2.6. Cytokine Analysis by Real-Time PCR. Mice skin was immediately frozen in liquid nitrogen and kept at $-70^{\circ} \mathrm{C}$ until use. For real-time PCR assay, mice skin was homogenized with Ultra-Turrax T10 (IKA labortechnik, Seoul, Korea) and RNA extraction was performed using TRIzol (Invitrogen life technologies, NY, USA). RNA content was measured using the NanoDrop ND-1000 spectrophotometer (NanoDrop Technologies Inc.). $1 \mathrm{~g}$ of total cellular RNA from each sample was reverse transcribed using cDNA synthesis kit (TaKaRa, Japan). Quantitative PCR was performed using SYBR green iMaster and a LightCycler 480 (Roche, Switzerland). 


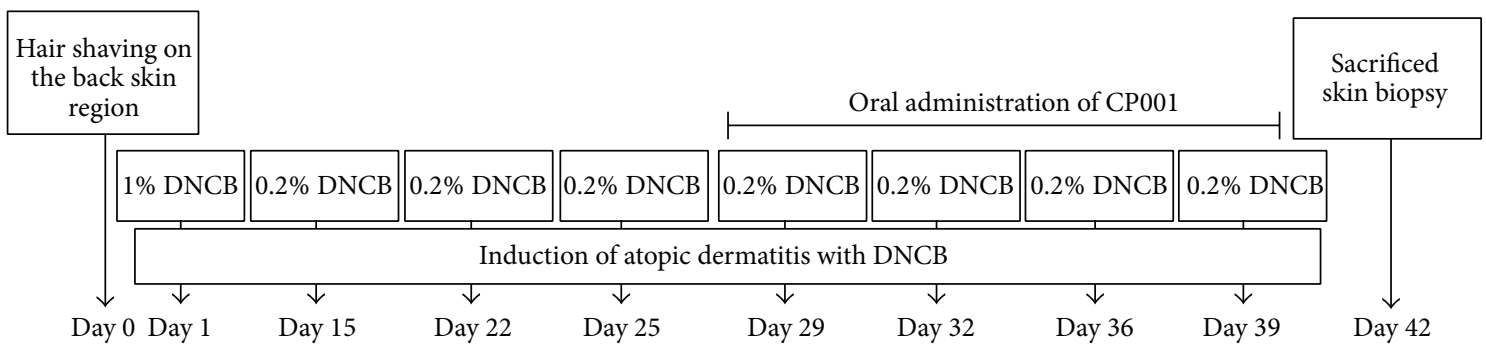

FIGURE 1: Protocols for induction of atopic dermatitis in mouse model. Shaved dorsal regions of the mice were sensitized with DNCB solution. Male BALB/c mice were epicutaneously sensitized with $200 \mu \mathrm{l}$ of a $1 \%$ DNCB solution on day 1 . Two weeks later, dermatitis was induced with $200 \mu \mathrm{l}$ of $0.2 \%$ DNCB solution at the intervals shown in the figure. CP001 was orally administrated from the 3rd week during sensitization together with DNCB.

TABLE 1

\begin{tabular}{ll}
\hline Primer name & Sequence $\left(5^{\prime}-3^{\prime}\right)$ \\
\hline \multirow{2}{*}{ IL-4 } & Forward: AAGAACACCACAGAGAGTGAGCTC \\
& Reverse: TTTCAGTGTGGACTTCCACTC \\
IL-13 & Forward: AGCATGGTATGGAGTGTGGACCTG \\
& Reverse: CAGTTGCTTTGTGTAGCTGAGCAG \\
IL-6 & Forward: AACCTTCCAAAGATGGCTGAA \\
& Reverse: CAGGAACTGGATCAGGACTTT \\
IL-8 & Forward: TCAGTGCATAAAGACATACTCC \\
& Reverse: TGGCATCTTCACTGATTCTTG \\
GAPDH & Forward: GAGGGGCCATCCACAGTCTTC \\
& Reverse: CATCACCATCTTCCAGGAGCG \\
\hline
\end{tabular}

2.7. Reverse Transcription-Polymerase Chain Reaction (RT$P C R)$. Cells were harvested by centrifugation and the pellet was washed with ice-cold PBS. RNA was isolated from the pellet using easy-blue RNA extraction kit (iNtRON Biotech, Republic of Korea) according to the manufacturer's instructions. Isolated RNA content was measured using the NanoDrop ND-1000 spectrophotometer (NanoDrop Technologies Inc.). $2 \mu \mathrm{g}$ of total cellular RNA from each sample was reverse transcribed using cDNA synthesis kit (TaKaRa, Japan). PCR was conducted out in a $20 \mu \mathrm{L}$ reaction mixture consisting of DNA template, $10 \mathrm{pM}$ of each gene-specific primer, 10x Taq buffer, $2.5 \mathrm{mM}$ dNTP mixture, and 1 unit of Taq DNA polymerase (Takara, Japan). PCR was performed using the specific primer and primer sequences for human IL-6, IL-8, and GAPDH are shown in Table 1.

2.8. HPLC Analysis. Ellagic acid, quercitrin hydrate, and catalpol were purchased from Sigma Chemicals (Saint Louis, $\mathrm{MO}$ ). Purity of standard compounds was guaranteed to be higher than $95 \%$ by HPLC. HPLC grade acetonitrile, methanol, and formic acid were purchased from J. T. Baker (Phillipsburg, NJ). Catalpol, ellagic acid, and quercitrin were chosen as Marker compounds to standardize the extract sample. CP001 was dissolved in distilled water at a concentration of $100 \mathrm{mg} / \mathrm{mL}$ and the solution was filtered through a $0.45 \mu \mathrm{m}$ membrane filter. A $10 \mu \mathrm{L}$ aliquot of the sample solution was injected into a HPLC system (Agilent Technologies, Palo
Alto, CA). The sample was analyzed on a Capcell Pak UG120 $\mathrm{C}_{18}$ analytical column $(250 \times 4.6 \mathrm{~mm}, 5 \mu \mathrm{m}$; Shiseido, Japan $)$.

2.9. Statistical Analysis. Statistical analyses presented as the mean \pm standard error of the mean (SEM) and were analyzed for statistical significance using the unpaired Student's $t$-test. $P$ value $<0.05$ was considered statistically significant.

\section{Results}

3.1. Oral Administration of CP001 Decreases Infiltration of Inflammatory Cells into AD-Like Skin Lesions. To determine whether CP001 decreases infiltration of inflammatory cells into $\mathrm{AD}$-like skin lesions, we performed $\mathrm{H} \& \mathrm{E}$ staining on the skin after oral administration of CP001. We observed infiltration of inflammatory cells into the epidermis and dermis in DNCB group, whereas CP001 decreased such infiltration of inflammatory cells into the skin (Figure 2). Moreover, CP001 (25-200 mg/kg) abrogated skin thickening induced by DNCB (Figure 2). Next, we also performed toluidine blue staining for mast cell observation. Repeated cutaneous application of DNCB increased dermal mast cell number. However, this feature was significantly suppressed by CP001 (Figure 3 ).

3.2. CP001 Administration Downregulates mRNA Expression of Th2 Cytokines. The Th2 type cytokines are important in an acute phase of $\mathrm{AD}$ whereas mixed Th2/Th1 type inflammation is characteristic to a chronic phase of $\mathrm{AD}$. To determine whether CP001 decreases Th2 type cytokines expression, we performed real-time PCR to measure IL-4 and IL-13 levels. We found that oral administration of CP001 decreased IL-4 mRNA expression in AD-like skin lesions (Figure 4(a)). We also found that CP001 administration decreased IL-13 mRNA expression in AD-like skin lesions in a dose-dependent manner (Figure 4(b)). In histology analysis, repeated cutaneous application of DNCB increased dermal mast cell number and this feature was suppressed by CP001 oral administration. Activated mast cells secrete various chemokines and cytokines including IL- 6 and IL8. To determine whether CP001 decreases IL-6 and IL-8 cytokines mRNA levels, we performed RT-PCR analysis in AD-like skin lesions. Oral administration of CP001 did not 

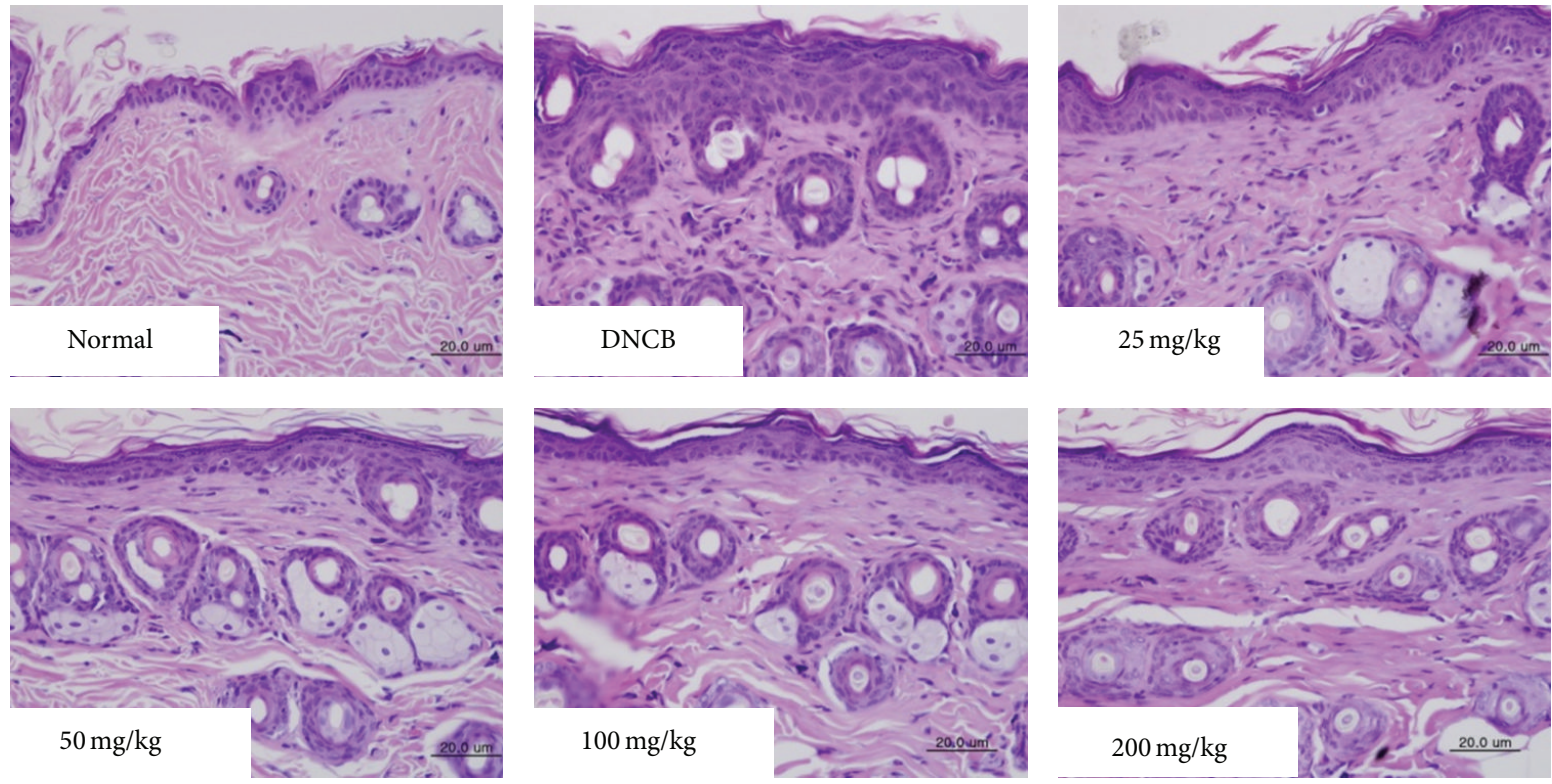

Figure 2: Histological features of AD-like skin lesions treated with CP001. The skin sections were stained with hematoxylin and eosin. Inflammatory cells infiltration into the dermis was measured after treatment with CP001 in the presence of DNCB. Sections were evaluated using microscope at an original magnification of 400x.

affect the suppression of IL-6 and IL- 8 mRNA expression in AD-like skin lesions (Figures 4(c) and 4(d)).

\subsection{CP001 Administration Downregulates Serum IgE Concen-} tration. Hyperproduction of $\mathrm{IgE}$ is a major characteristic of $\mathrm{AD}$ and patients with $\mathrm{AD}$ often exhibit elevated levels of total and allergen specific IgE antibodies (Abs) in their serum. To further test whether suppression of the progression of $\mathrm{AD}$ like skin lesions by CP001 is associated with serum IgE levels, we performed total IgE ELISA assay. We found that total IgE levels were dramatically elevated in DNCB-treated group compared with normal group. However, increased serum IgE levels induced by DNCB were significantly decreased by CP001 treatment (Figure 5).

\subsection{Effect of CP001 on PMA Plus A23187-Induced IL-6 and IL-} 8 Expression in HMC-1. Next, we investigated whether CP001 affects production of IL- 6 and IL-8 in HMC-1. For that purpose, mast cells were pretreated with various concentrations of CP001 for $1 \mathrm{~h}$ and then treated with PMA and A23187 for $24 \mathrm{~h}$. The levels of IL- 6 and IL- 8 in culture supernatants were measured by ELISA assay. We found that IL- 6 secretion induced by PMA and A23187 was significantly suppressed by CP001 (Figures 6(a) and 6(c)). We also performed RT-PCR to measure IL-6 and IL-8 mRNA expression in HMC-1. We observed that IL- 6 and IL- 8 mRNA induced by PMA and A23187 were decreased by CP001 (Figures 6(b) and 6(d)).

3.5. Effect of CP001 on PMA Plus A23187-Induced Th2 Cytokine Expression in HMC-1. CP001 administration decreased IL-4 and IL-13 mRNA expression in AD-like skin lesions (Figure 7(b)). Therefore, we characterized the regulatory effect of CP001 on IL-4 and IL-13 mRNA expression in HMC-1 using RT-PCR. We found that IL-13 expression induced by PMA and A23187 was significantly suppressed by CP001 (Figure 7(a)). IL-4 expression level was not increased by PMA and A23187, but it is suppressed by CP001 (Figure 7(a)).

3.6. HPLC Analysis. To further evaluate the effective compounds of CP001 extract, HPLC analysis was employed. In order to analyze catalpol, the mobile phase consisted of water (W) and methanol (M) and the flow rate was $1 \mathrm{~mL} / \mathrm{min}$. The gradient elution program was used as follows. The initial composition of the mobile phase was $97: 3$ (W:M), which was linearly changed to $95: 5$ (W:M) over $1 \mathrm{~min}$ and changed to $91: 9(\mathrm{~W}: \mathrm{M})$ for $9 \mathrm{~min}$. At $11 \mathrm{~min}$, the composition of mobile phase returned to the initial condition, which was maintained for $9 \mathrm{~min}$ for column reequilibration. Chromatograms were acquired at $210 \mathrm{~nm}$ by UV detection (Figure 8). For ellagic acid and quercitrin, the mobile phase consisted of $0.1 \%$ formic acid (F) and acetonitrile (A) and the flow rate was $1 \mathrm{~mL} / \mathrm{min}$. The gradient elution program was used as follows. The initial composition of the mobile phase was $90: 10$ (F:A), which was linearly changed to $85: 15$ (F:A) over $5 \mathrm{~min}$ and changed to $60: 40$ (F:A) for $35 \mathrm{~min}$. At $41 \mathrm{~min}$, the composition of mobile phase returned to the initial condition, which was maintained for $9 \mathrm{~min}$ for column reequilibration. Chromatograms were acquired at $254 \mathrm{~nm}$ by UV detection. The retention times of catalpol, ellagic acid, and quercitrin were $6.2,14.4$, and $18.6 \mathrm{~min}$, respectively (Figures 7(a) and 7(b)). The concentrations of catalpol, ellagic acid, and quercitrin in the extract sample were determined using HPLC analysis as described above. The extract was standardized to contain $1.8 \%$ catalpol, $0.4 \%$ ellagic acid, and $0.3 \%$ quercitrin. 

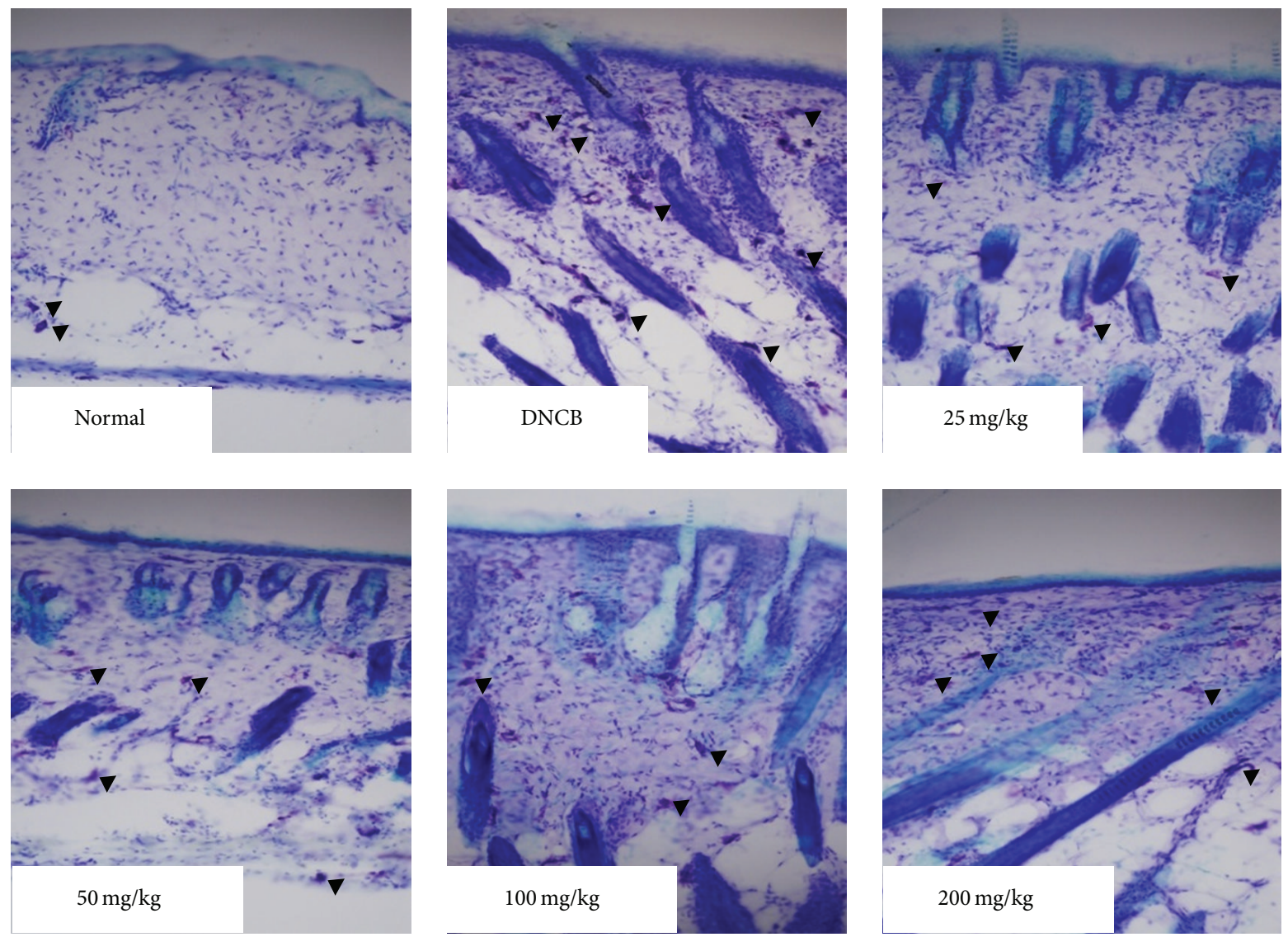

(a)

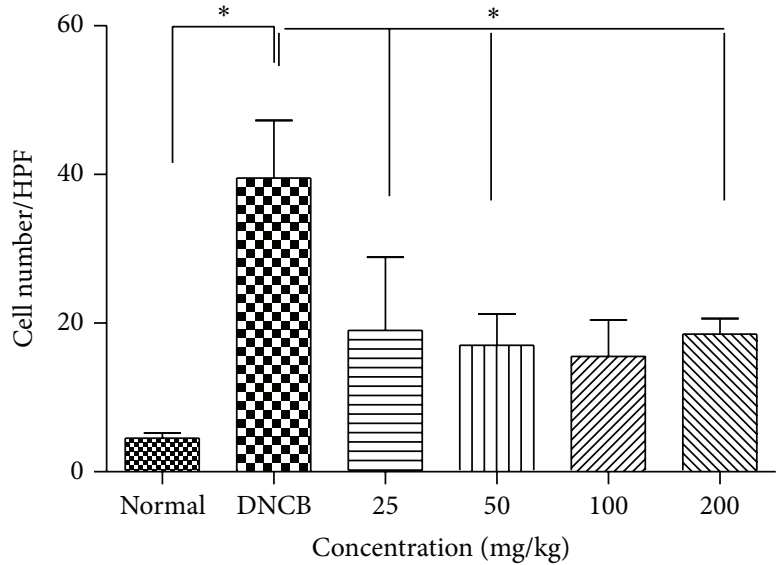

(b)

FIGURE 3: The measurement of mast cells number in AD-like skin lesions treated with CP001. The skin sections were stained with toluidine blue for mast cells staining. Sections were evaluated using microscope at an original magnification of 400x. The data are presented as mean \pm SD from five animals in each group. ${ }^{*} P<0.05$.

\section{Discussion}

$\mathrm{AD}$ is a chronic inflammatory disease, which is accompanied by erythema, edema, and scaling in AD skin lesions [24]. Recently, Korean medicine has been the subject of increased interest for its potential in the treatment of inflammatory diseases, including atopic dermatitis and airway inflammation $[16,17,25,26]$. The present study demonstrates that oral administration of Korean herbal mixture extract, CP001, inhibits DNCB-induced AD. We observed that CP001 decreases infiltration of inflammatory cells into AD-like skin lesions and dermal mast cell number.

Generally, steroid therapy is used for AD treatment, but it cannot be administrated over the long term because of 


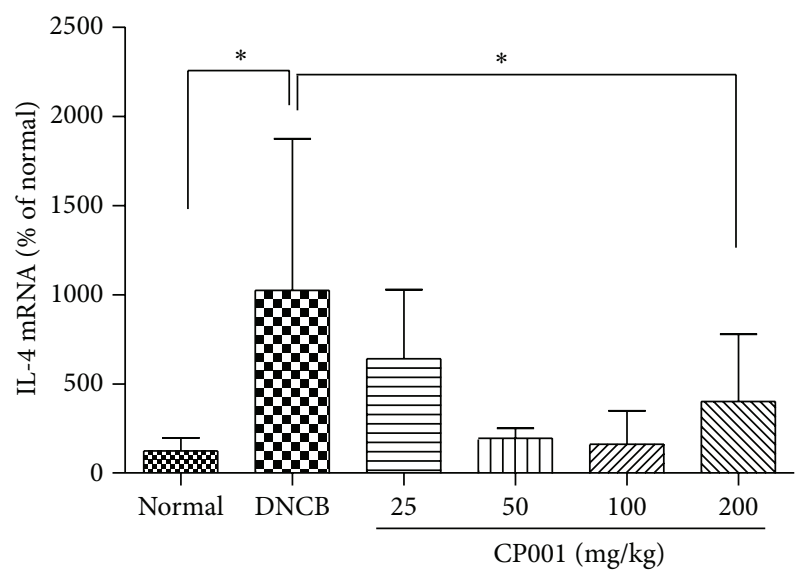

(a)

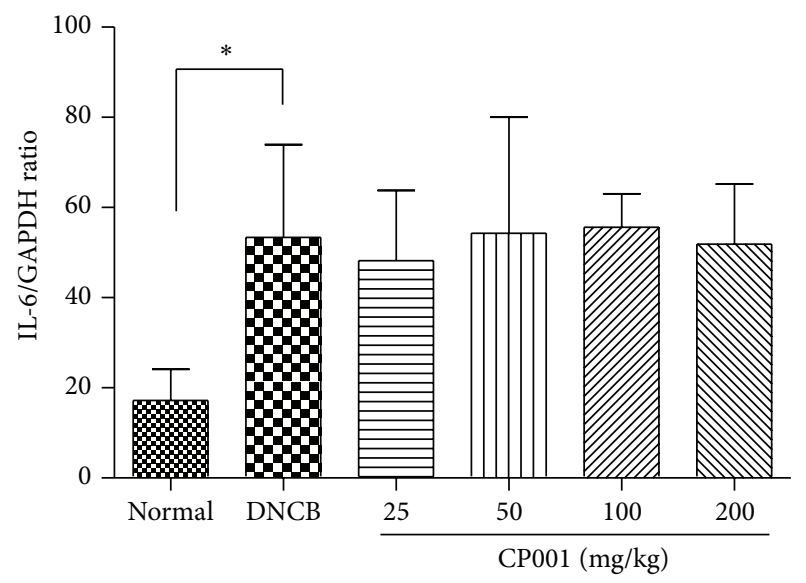

(c)

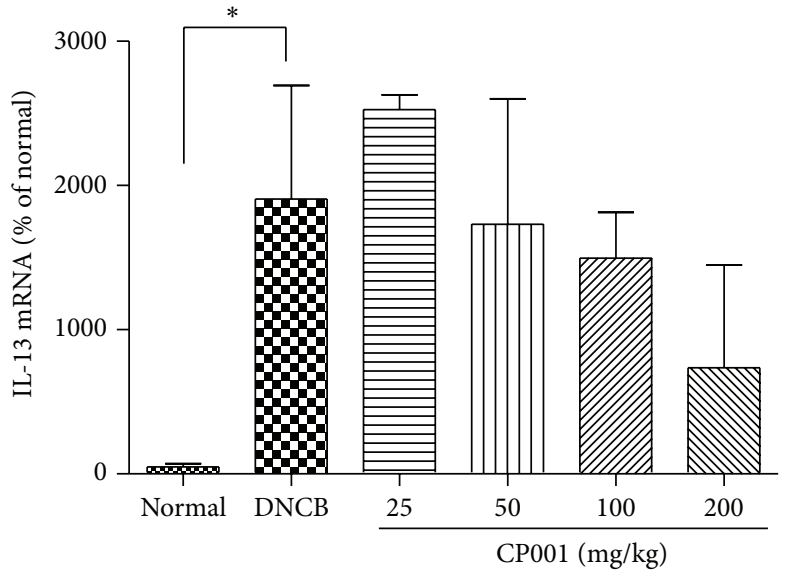

(b)

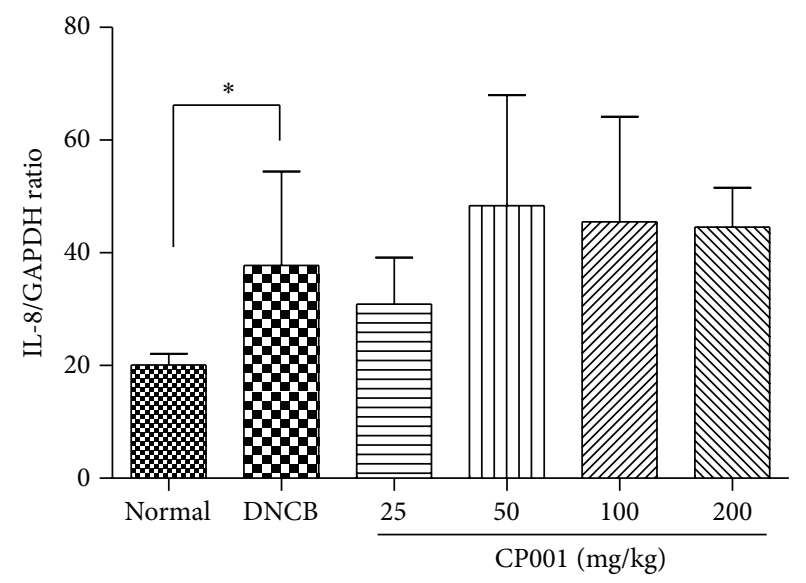

(d)

FIGURE 4: Effect of CP001 on the cytokine mRNA expression in mouse skin tissue. The IL-4, IL-13, IL6, and IL-8 mRNA expression were measured by real-time PCR (a), (b) and RT-PCR (c), (d) in mouse skin tissue. The columns and the error bars represent mean \pm SD $(n=5$ mice/group). ${ }^{*} P<0.05$.

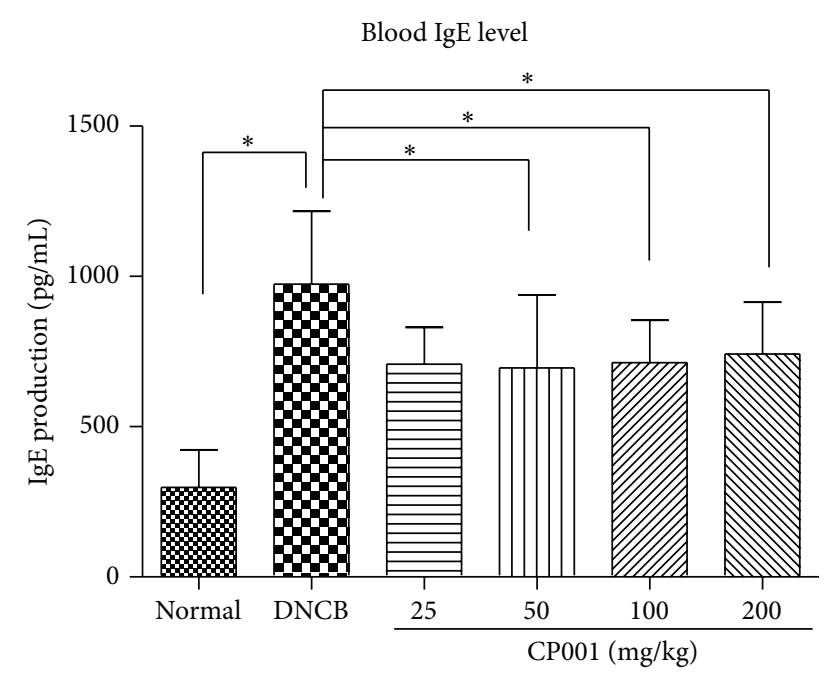

FIGURE 5: Measurement of plasma IgE level. Total IgE level was determined by ELISA. The columns and the error bars represent mean \pm SEMs ( $n=4$ mice/group). ${ }^{*} P<0.05$. the many side effects. Therefore, we find a new drug, which is effective in the treatment of $\mathrm{AD}$ without any side effects. Recently, we reported that topical application of KM110329 (CP001 modified drug) reduced ovalbumin- and DNCBinduced atopic dermatitis [23]. Therefore, we were wondering whether CP001 oral administration may inhibit DNCBinduced atopic dermatitis.

Mast cells degranulation can be regulated by the recruitment, trafficking, and function of inflammatory response. For example, IL-4 and IL-13 induce cell adhesion molecules on endothelium which can be recruitment of leukocytes [27-29]. Also, the production of IL-4 cytokine in epidermal cells has been known to be the main factor for initiation of $\mathrm{AD}$ [30]. In our data, we show that cytokine production and mRNA levels of IL-4, IL-13, IL-6, and IL-8 were suppressed by CP001 in HMC-1. Also, quantitative real-time PCR of the skin lesions also showed that oral administration of CP001 diminished the mRNA level of IL-4 and IL-13 in the AD-like skin lesions. In addition, we found that CP001 reduces mast cell in 


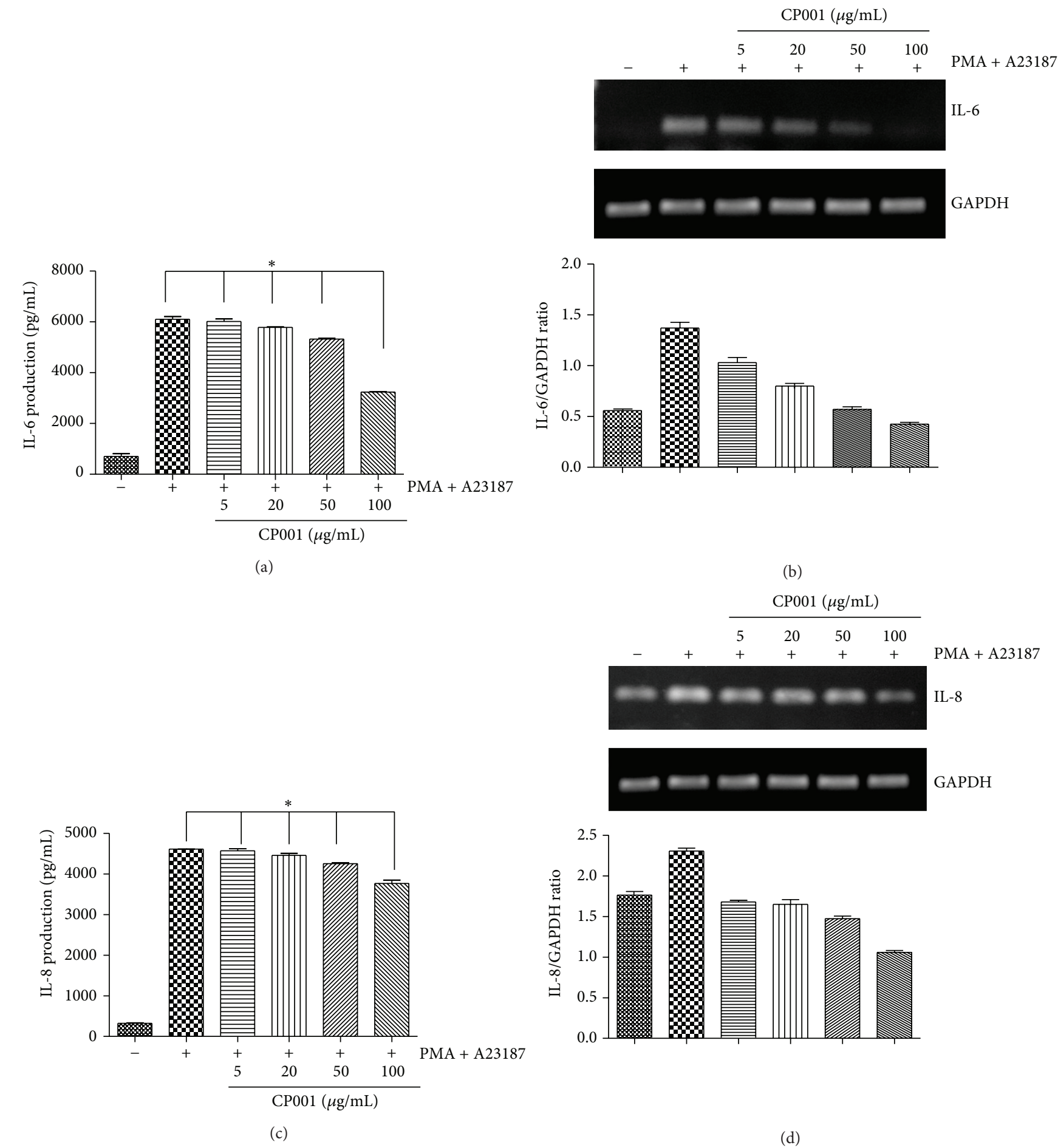

FIGURE 6: Effect of CP001 on PMA plus A23187-stimulated proinflammatory cytokine expression in HMC-1. HMC-1 were pretreated with various concentrations of CP001 for $1 \mathrm{~h}$ and then treated with PMA and A23187 for $24 \mathrm{~h}$. The levels of IL- 6 and IL- 8 in culture supernatants were measured by ELISA assay (a), (c). The IL- 6 and IL- 8 mRNA levels were measured by RT-PCR (b), (d). Data represent the mean \pm SEMs of three independent experiments. 

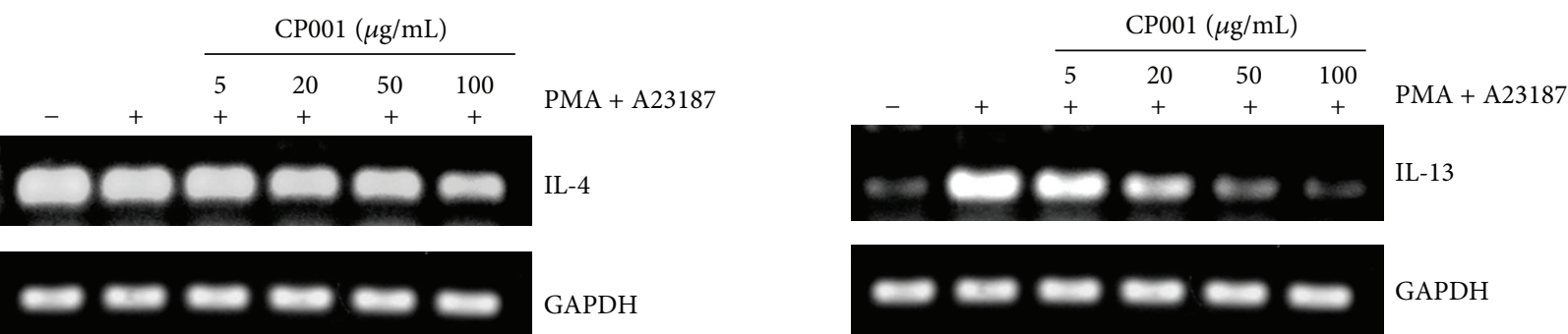

GAPDH
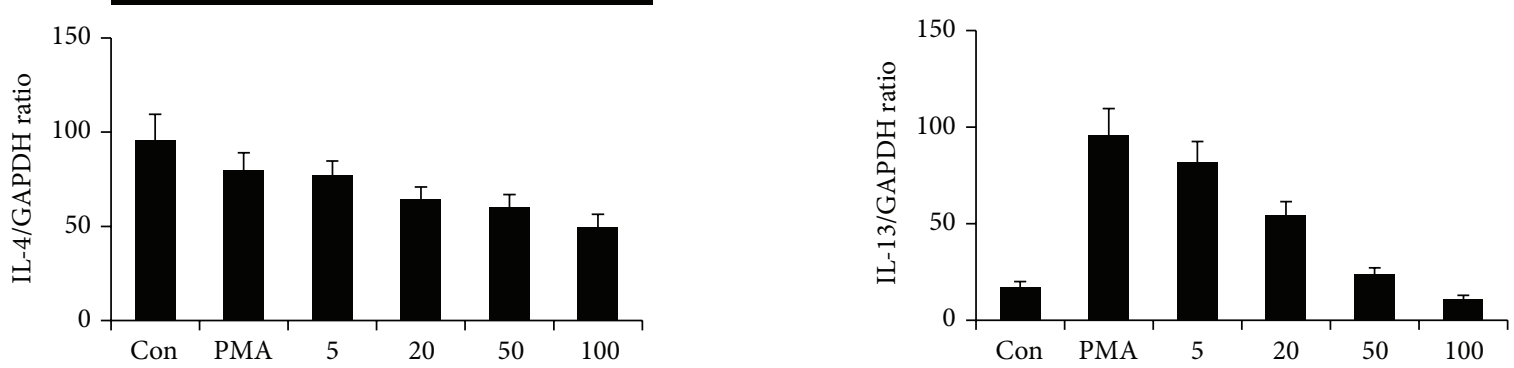

(a)

(b)

FIGURE 7: Effect of CP001 on PMA plus A23187-stimulated Th2 cytokine expression in HMC-1. HMC-1 were pretreated with various concentrations of CP001 for $1 \mathrm{~h}$ and then treated with PMA and A23187 for $24 \mathrm{~h}$. The IL- 4 and IL-13 mRNA levels were measured by RTPCR (a), (b). Data represent the mean \pm SEMs of three independent experiments.

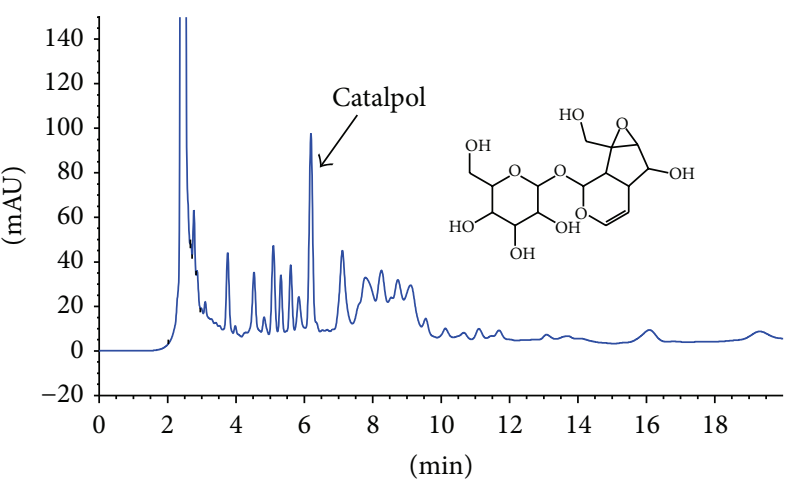

(a)

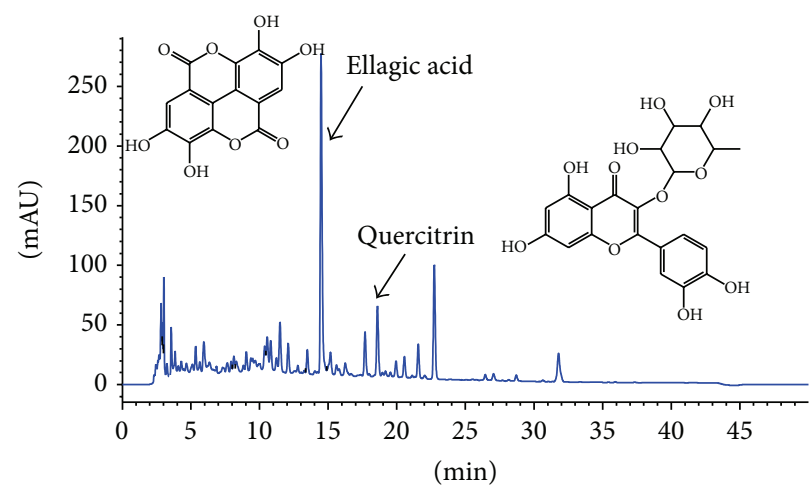

(b)

FIGURE 8: Typical HPLC chromatograms of CP001 for (a) catalpol, (b) ellagic acid, and quercitrin.

DNCB-induced AD-like skin lesion. It seems that inhibition of infiltration of mast cell downregulates secretion of IL- 4 and IL-13 cytokines and it may inhibit recruitment of leukocytes. Thus, mast cell may be main factor for suppression of Th2 cytokines in the AD-like skin lesions by oral administration of CP001.

$\operatorname{IgE}$ is mediator of mast cell activation and we observed that CP001 oral administration reduced elevated blood IgE levels induced by repeated DNCB sensitization

CP001 also suppressed IL-6 secretion and elevated IL-6 and IL-8 mRNA expression induced by PMA and A23187 in HMC-1. It seems that the reduction of infiltration of mast cells is related to decrease of degranulation of mast cells and maturation of eosinophils suppressing the release of various inflammatory cytokines.

\section{Conclusion}

Our present study clearly demonstrates that CP001 suppresses the progression of $\mathrm{AD}$ induced by DNBC. In addition, inflammatory related cytokine production and mRNA levels of IL-4, IL-13, IL-6, and IL-8 were suppressed by CP001. This suggests that CP001 might be a useful candidate for the treatment of AD.

\section{Conflict of Interests}

The authors declare that there is no conflict of interests regarding the publication of this paper.

\section{Authors' Contribution}

Soon Re Kim and Han-Seok Choi contributed equally to this study. 


\section{Acknowledgment}

This study was supported by a Grant of the Traditional Korean Medicine R \& D Project of the Ministry for Health \& Welfare, Republic of Korea (B110017 and HI12C1889).

\section{References}

[1] C. A. Akdis, M. Akdis, T. Bieber et al., "Diagnosis and treatment of atopic dermatitis in children and adults: European Academy of Allergology and Clinical Immunology/American Academy of Allergy, Asthma and Immunology/PRACTALL consensus report," Allergy: European Journal of Allergy and Clinical Immunology, vol. 118, no. 1, pp. 152-169, 2006.

[2] D. Y. Leung, M. Boguniewicz, M. D. Howell, I. Nomura, and Q. A. Hamid, "New insights into atopic dermatitis," The Journal of Clinical Investigation, vol. 113, no. 5, pp. 651-657, 2004.

[3] S. P. Stone, G. J. Gleich, and S. A. Muller, "Atopic dermatitis and IgE: relationship between changes in IgE levels and severity of disease," Archives of Dermatology, vol. 112, no. 9, pp. 1254-1255, 1976.

[4] S. J. Galli and M. Tsai, "IgE and mast cells in allergic disease," Nature Medicine, vol. 18, no. 5, pp. 693-704, 2012.

[5] H. Matsuda, N. Watanabe, G. P. Geba et al., "Development of atopic dermatitis-like skin lesion with IgE hyperproduction in NC/Nga mice," International Immunology, vol. 9, no. 3, pp. 461466, 1997.

[6] E. B. Brandt and U. Sivaprasad, "Th2 cytokines and atopic dermatitis," Journal of Clinical Cell Immunology, vol. 2, no. 3, p. 110, 2011.

[7] P. Bradding, I. H. Feather, S. Wilson et al., "Immunolocalization of cytokines in the nasal mucosa of normal and perennial rhinitic subjects: the mast cell as a source of IL-4, IL-5, and IL- 6 in human allergic mucosal inflammation," The Journal of Immunology, vol. 151, no. 7, pp. 3853-3865, 1993.

[8] J. A. M. Mican, N. Arora, P. R. Burd, and D. D. Metcalfe, "Passive cutaneous anaphylaxis in mouse skin is associated with local accumulation of interleukin-6 mRNA and immunoreactive interleukin-6 protein," Journal of Allergy and Clinical Immunology, vol. 90, no. 5, pp. 815-824, 1992.

[9] P. Salamon, N. G. Shoham, R. Gavrieli, B. Wolach, and Y. A. Mekori, "Human mast cells release Interleukin-8 and induce neutrophil chemotaxis on contact with activated T cells," Allergy, vol. 60, no. 10, pp. 1316-1319, 2005.

[10] A. Moller, U. Lippert, D. Lessmann et al., "Human mast cells produce IL-8," Journal of Immunology, vol. 151, no. 6, pp. 32613266, 1993.

[11] T.-Y. Lin, Y.-C. Liu, J.-R. Jheng et al., "Anti-enterovirus 71 activity screening of Chinese herbs with anti-infection and inflammation activities," The American Journal of Chinese Medicine, vol. 37, no. 1, pp. 143-158, 2009.

[12] Y. Tang, J. Yang, C. Lin et al., "Houttuynia cordata thunb extract induces apoptosis through mitochondrial-dependent pathway in HT-29 human colon adenocarcinoma cells," Oncology Reports, vol. 22, no. 5, pp. 1051-1056, 2009.

[13] R. X. Zhang, M. X. Li, and Z. P. Jia, "Rehmannia glutinosa: review of botany, chemistry and pharmacology," Journal of Ethnopharmacology, vol. 117, no. 2, pp. 199-214, 2008.

[14] Y. Y. Sung, T. Yoon, J. Y. Jang, S. J. Park, and H. K. Kim, "Topical application of Rehmannia glutinosa extract inhibits mite allergen-induced atopic dermatitis in NC/Nga mice," Journal of Ethnopharmacology, vol. 134, no. 1, pp. 37-44, 2011.
[15] P. S. Wu, S. J. Wu, Y. H. Tsai, Y. H. Lin, and J. C. Chao, "Hot water extracted Lycium barbarum and Rehmannia glutinosa inhibit liver inflammation and fibrosis in rats," The American Journal of Chinese Medicine, vol. 39, no. 6, pp. 1173-1191, 2011.

[16] H. Kim, E. Lee, S. Lee, T. Shin, Y. Kim, and J. Kim, "Effect of Rehmannia glutinosa on immediate type allergic reaction," International Journal of Immunopharmacology, vol. 20, no. 4-5, pp. 231-240, 1998.

[17] H. M. Kim, C. S. An, K. Y. Jung, Y. Choo, J. Park, and S. Nam, "Rehmannia glutinosa inhibits tumour necrosis factor- $\alpha$ and interleukin-1 secretion from mouse astrocytes," Pharmacological Research, vol. 40, no. 2, pp. 171-176, 1999.

[18] B. Jung, S. Cho, H. Koh, D. Han, and B. Lee, "Fermented Maesil (Prunus mume) with probiotics inhibits development of atopic dermatitis-like skin lesions in NC/Nga mice," Veterinary Dermatology, vol. 21, no. 2, pp. 184-191, 2010.

[19] E. Kim, H. Lee, S. K. Kim et al., "The bark of Betula platyphylla var. japonica inhibits the development of atopic dermatitis-like skin lesions in NC/Nga mice," Journal of Ethnopharmacology, vol. 116, no. 2, pp. 270-278, 2008.

[20] J.-E. Huh, J.-M. Hong, Y.-H. Baek, J.-D. Lee, D.-Y. Choi, and D.-S. Park, "Anti-inflammatory and anti-nociceptive effect of Betula platyphylla var. japonica in human interleukin-1 $\beta$ stimulated fibroblast-like synoviocytes and in experimental animal models," Journal of Ethnopharmacology, vol. 135, no. 1, pp. 126-134, 2011.

[21] J. Y. Bae, S. S. Lim, J. S. Choi, and Y. H. Kang, "Protective actions of Rubus coreanus ethanol extract on collagenous extracellular matrix in ultraviolet-B irradiation-induced human dermal fibroblasts," Nutritiona Research and Practice, vol. 1, no. 4, pp. 279-284, 2007.

[22] S. H. Ko, S. W. Choi, and S. K. Ye, "Comparison of antioxidant activities of seventy herbs that have been used in Korean traditional medicine," Nutritiona Research and Practice, vol. 2, no. 3, pp. 143-151, 2008.

[23] S. R. Kim, H. Choi, H. S. Seo, Y. K. Choi, Y. C. Shin, and S. Ko, "Topical application of herbal mixture extract inhibits ovalbumin- or 2,4-dinitrochlorobenzene-induced atopic dermatitis," Evidence-Based Complementary and Alternative Medicine, vol. 2012, Article ID 545497, 9 pages, 2012.

[24] N. I. Denig, A. W. Hoke, and H. I. Maibach, "Irritant contact dermatitis. Clues to causes, clinical characteristics, and control," Postgraduate Medicine, vol. 103, no. 5, pp. 199-200, 207-208, 212213, 1998.

[25] J. H. Lee and S. H. Cho, "Korean red ginseng extract ameliorates skin lesions in NC/Nga mice: an atopic dermatitis model," Journal of Ethnopharmacology, vol. 133, no. 2, pp. 810-817, 2011.

[26] Z. Quan, Y. J. Lee, J. H. Yang et al., "Ethanol extracts of Saururus chinensis suppress ovalbumin-sensitization airway inflammation," Journal of Ethnopharmacology, vol. 132, no. 1, pp. 143-149, 2010.

[27] L. J. Walsh, G. Trinchieri, H. A. Waldorf, D. Whitaker, and G. F. Murphy, "Human dermal mast cells contain and release tumor necrosis factor $\alpha$, which induces endothelial leukocyte adhesion molecule 1," Proceedings of the National Academy of Sciences of the United States of America, vol. 88, no. 10, pp. 4220-4224, 1991.

[28] R. P. Schleimer, S. A. Sterbinsky, J. Kaiser et al., "IL-4 induces adherence of human eosinophils and basophils but not neutrophils to endothelium: association with expression of VCAM1," Journal of Immunology, vol. 148, no. 4, pp. 1086-1092, 1992.

[29] M. Sironi, F. L. Sciacca, C. Matteucci et al., "Regulation of endothelial and mesothelial cell function by interleukin-13: 
selective induction of vascular cell adhesion molecule- 1 and amplification of interleukin-6 production," Blood, vol. 84, no. 6, pp. 1913-1921, 1994.

[30] L. S. Chan, N. Robinson, and L. Xu, "Expression of interleukin4 in the epidermis of transgenic mice results in a pruritic inflammatory skin disease: an experimental animal model to study atopic dermatitis," Journal of Investigative Dermatology, vol. 117, no. 4, pp. 977-983, 2001. 


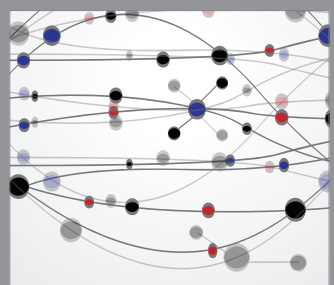

The Scientific World Journal
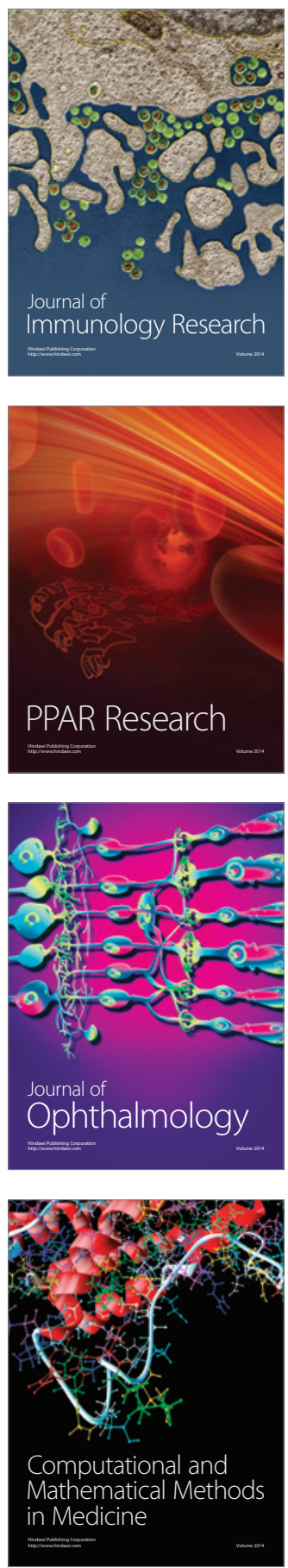

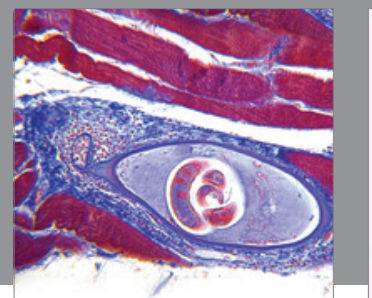

Gastroenterology

Research and Practice
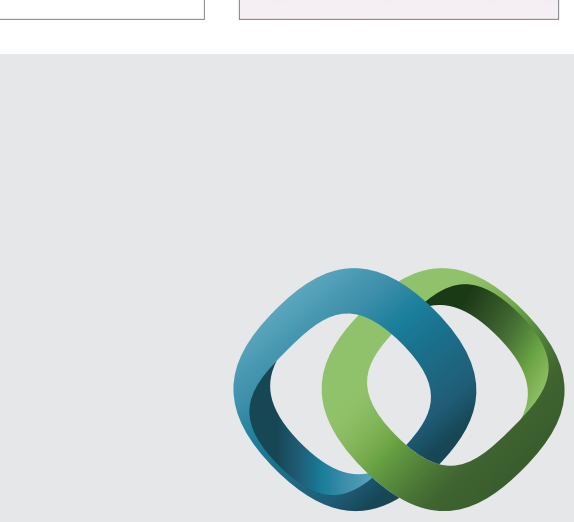

\section{Hindawi}

Submit your manuscripts at

http://www.hindawi.com
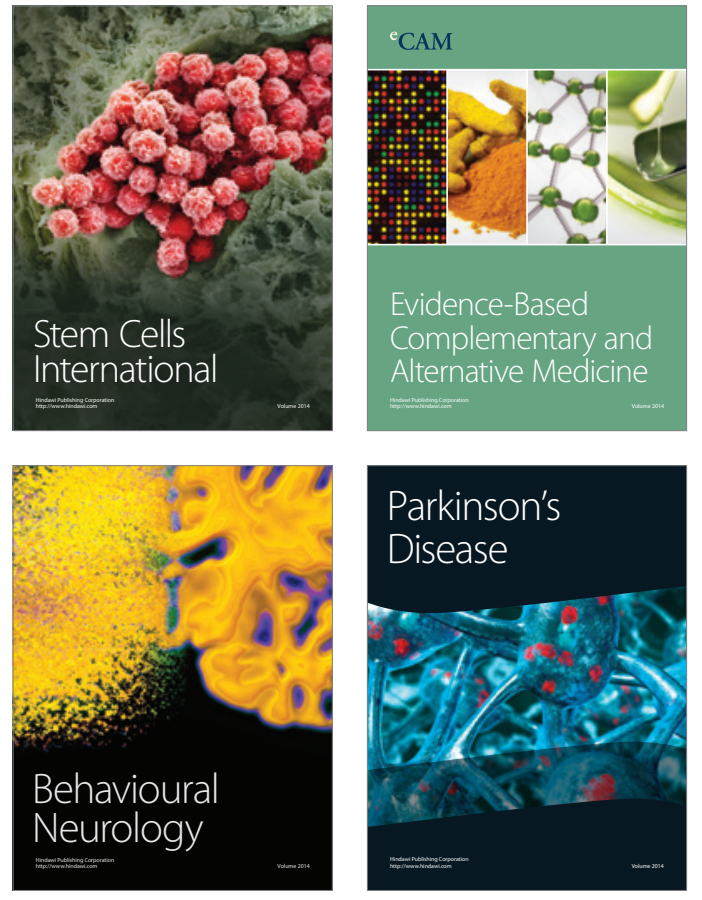
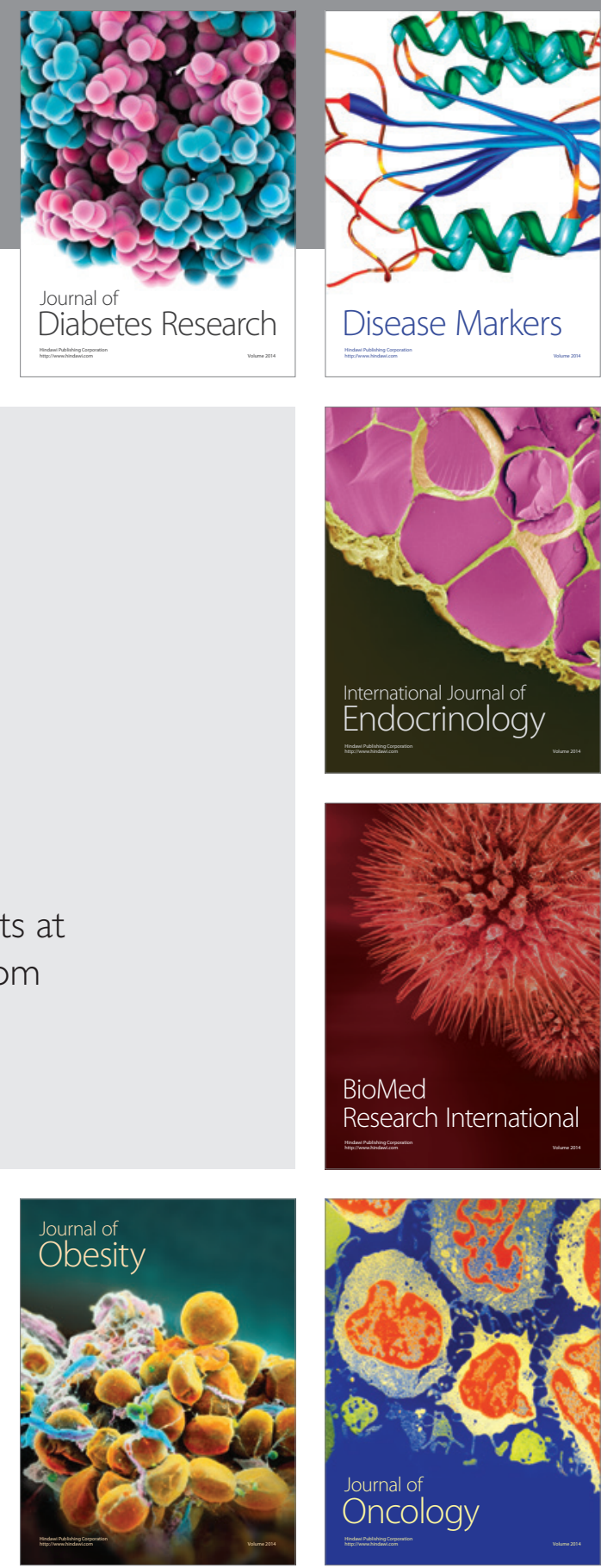

Disease Markers
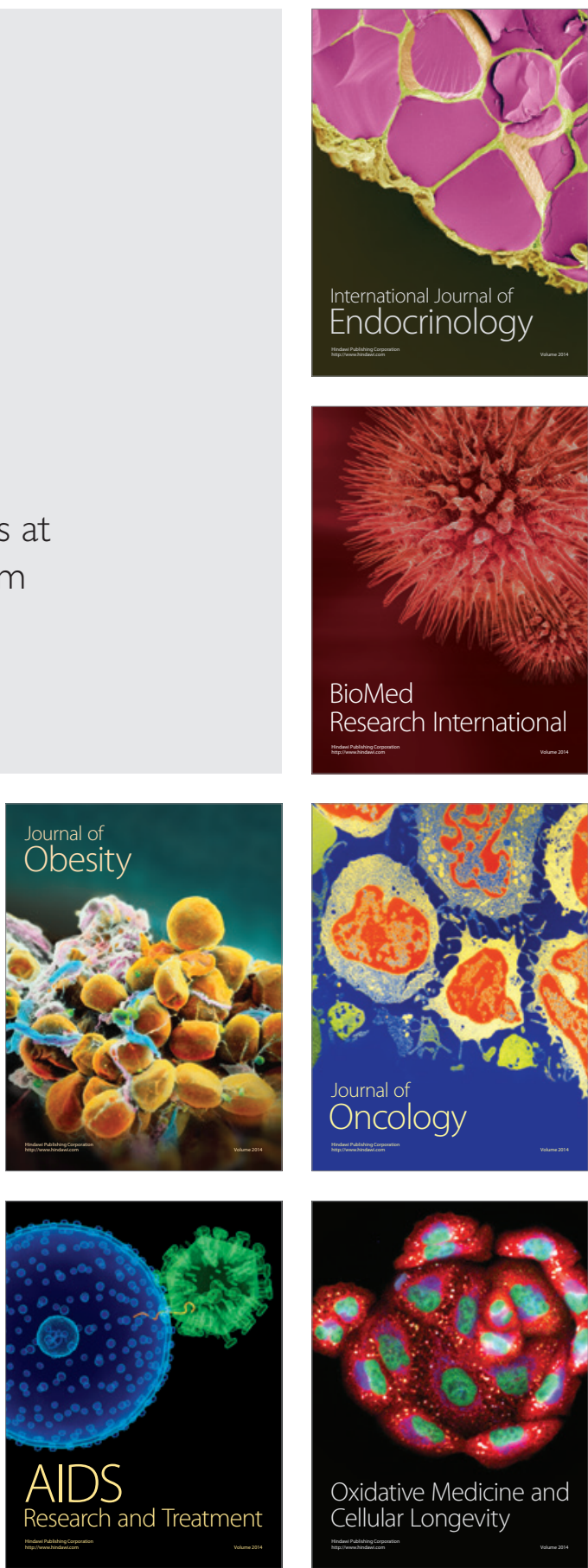